\title{
STRONG RAMSEY THEOREMS FOR STEINER SYSTEMS
}

\author{
JAROSLAV NEŠETŘIL AND VOJTĚCH RÖDL
}

\begin{abstract}
It is shown that the class of partial Steiner $(k, l)$-systems has the edge Ramsey property, i.e., we prove that for every partial Steiner $(k, l)$-system $\mathcal{G}$ there exists a partial Steiner $(k, l)$-system $\forall$ such that for every partition of the edges of $\mathcal{H}$ into two classes one can find an induced monochromatic copy of $\mathcal{G}$. As an application we get that the class of all graphs without cycles of lengths 3 and 4 has the edge Ramsey property. This solves a longstanding problem in the area.
\end{abstract}

1. Introduction, basic notions and the main theorem. Let $A$ be a class of $k$-uniform hypergraphs. $A$ is said to have the edge partition (or Ramsey) property if for every $H_{1} \in A$ and every positive integer $r$ there exists $\not_{2} \in A$ with the property that for every partition of the edges $\mathcal{E}\left(\mathscr{H}_{2}\right)=\mathcal{E}_{1} \cup \mathcal{E}_{2} \cup \cdots \cup \mathcal{E}_{r}$ there exists an induced subhypergraph $H_{1}^{\prime}$ of $H_{2}$ which is isomorphic to $H_{1}$ (i.e. $H_{1}^{\prime} \cong H_{1}$ ) and $i \in\{1,2, \ldots, r\}$ such that all edges of $\mathcal{H}_{1}^{\prime}$ are elements of $\mathcal{E}_{1}$.

The edge partition properties of many classes of graphs and hypergraphs were investigated in various papers of the present authors and others (cf. $[4,2,15,6,8-$ $11,1,13])$. In this paper we answer one of the longstanding problems of this area and show that the class of all partial Steiner $(k, l)$-systems has the edge partition property. This answers a problem raised in [3 and $\mathbf{7}]$ (see also [5]).

Our method is based on a refinement of the "partite amalgamation" which was used in [10-12]. Note that we deal with finite objects only.

A partial Steiner $(k, l)$-system $\mathcal{G}=(V, \mathcal{E})$ is a $k$-uniform hypergraph i.e. a system $\mathcal{E}$ of $k$-element subsets (edges) of a finite set $V_{1}$ which moreover has the property that ever $l$-element set is contained in at most one edge of $\mathcal{G}$. Our approach is based on the notion of partite hypergraph. Let $\left(V_{i}\right)_{i=1}^{m}$ be a system of pairwise disjoint sets and let $\mathcal{E}$ be a system of subsets of $\bigcup_{i=1}^{m} V_{i}$ with the property that $\left|E \cap V_{i}\right| \leq 1$ for every $i=1,2, \ldots, m$ and every $E \in \mathcal{E}$. Then the couple $\mathcal{G}=\left(\left(V_{i}\right)_{i=1}^{m}, \mathcal{E}\right)$ is called an $m$-partite hypergraph. Sometimes we write also $V_{i}=V_{i}(\mathcal{G})$ and $\mathcal{E}=\mathcal{E}(\mathcal{G})$.

Let $l \leq k$. By the symbol $\operatorname{Part}(k, l)$ we will denote the class of all $k$-partite $k$-uniform hypergraphs with the property that intersection of any two edges has cardinality smaller than $l$.

Let $\mathcal{G}=\left(\left(V_{i}\right)_{i=1}^{m}, \mathcal{E}\right), \mathcal{H}=\left(\left(W_{i}\right)_{i=1}^{n}, \mathcal{F}\right)$ be two partite hypergraphs. We say that the mapping $\phi: \bigcup_{i=1}^{m} V_{i} \rightarrow \bigcup_{j=1}^{n} W_{j}$ is a partite embedding if

(a) For every $i=1,2, \ldots, m$ there exists $j_{i}=1,2, \ldots, n$ such that $\phi\left(V_{i}\right) \subset W_{j_{i}}$.

Received by the editors August 20, 1986.

1980 Mathematics Subject Classification (1985 Revision). Primary 05C65, 05C55; Secondary $05 \mathrm{C} 15$. 
(b) $\phi$ is an embedding of $\left(\bigcup_{i=1}^{m} V_{i}, \mathcal{E}\right)$ into $\left(\bigcup_{i=1}^{n} W_{i}, \mathcal{F}\right)$ i.e.

(i) $\phi$ is $1-1$ and

(ii) $\left\{\phi\left(\nu_{1}\right), \ldots, \phi\left(\nu_{k}\right)\right\} \in \mathcal{F}$ if and only if $\left\{\nu_{1}, \nu_{2}, \ldots, \nu_{k}\right\} \in \mathcal{E}$.

We further say that $\mathcal{G}$ and $\mathcal{H}$ are isomorphic $(\mathcal{G} \cong \mathcal{H})$, if there exists a partite embedding

$$
\phi: \bigcup_{i=1}^{m} V_{i} \rightarrow \bigcup_{j=1}^{n} W_{j}
$$

which is onto. (This of course implies that $m=n$ and $\left|V_{i}\right|=\left|W_{j_{i}}\right|$ ). We say that $\mathcal{G}=\left(\bigcup_{i=1}^{m} V_{i}, \mathcal{E}\right)$ is an induced partite subgraph of $\mathcal{H}=\left(\bigcup_{i=1}^{n} W_{i}, \mathcal{F}\right)$ if $\bigcup_{i=1}^{m} V_{i} \subset$ $\bigcup_{j=1}^{n} W_{j}$ and the identity map

$$
\iota: \bigcup_{i=1}^{m} V_{i} \rightarrow \bigcup_{j=1}^{n} W_{j}
$$

is a partite embedding. This fact will be abbreviated by $\mathcal{G} \leq \mathcal{H}$. The set of all induced partite subgraphs of a partite hypergraph $\mathcal{H}=\left(\left(W_{i}\right)_{i=1}^{n}, \mathcal{F}\right)$, which are isomorphic to $\mathcal{G}=\left(\left(V_{i}\right)_{i=1}^{m}, \mathcal{E}\right)$ is denoted by $\left(\begin{array}{l}\mathcal{H} \\ \mathcal{G}\end{array}\right)$. If $\mathcal{G}$ is a hypergraph, then $V(\mathcal{G})$ denotes the vertex set and $\mathcal{E}(\mathcal{G})$ the edge set of hypergraph $\mathcal{G}$. If $\mathcal{G}$ and $\mathcal{H}$ are two hypergraphs, we say that $\mathcal{G}$ is a subgraph of $\not H$ if $V(\mathcal{G}) \subset V(\mathcal{H})$ and $\mathcal{E}(\mathcal{G}) \subset \mathcal{E}(\mathcal{H})$. We say that $\mathcal{G}$ is an induced subgraph if in addition $\mathcal{E}(\mathcal{G})=[V(\mathcal{G})]^{k} \cap \mathcal{E}(\mathcal{G})$ holds, where $[V(\mathcal{G})]^{k}$ is the set of $k$-tuples of the set $V(\mathcal{G})$. The complete $k$-uniform hypergraph $K_{m}^{k}$ is the hypergraph with $m$-element vertex set $V$ and edge set $[V]^{k}$.

The main objective of this paper is to prove the following.

THEOREM 1.1. The class of all partial Steiner $(k, l)$-systems has the edge partition property i.e. more explicitly for every partial Steiner $(k, l)$-system $\mathcal{G}$ and every positive integer $r$ there exists a partial Steiner $(k, l)$-system $\mathcal{H}=(W, \mathcal{F})$ with the following property: if the edge set of $H$ is partitioned into $r$ classes in an arbitrary way $\mathcal{F}=\mathcal{F}_{1} \cup \mathcal{F}_{2} \cup \cdots \cup \mathcal{F}_{r}$ then there exists $i \leq r$ and an induced subhypergraph $\mathcal{G}^{\prime}$ of $\mathcal{H}, \mathcal{G}^{\prime}$ isomorphic to $\mathcal{G}$ so that all edges of $\mathcal{G}^{\prime}$ are elements of $\mathcal{F}_{i}$.

The partition property of the hypergraph $\mathcal{H}$, described in the above theorem will be sometimes abbreviated by the symbol $\mathcal{H} \rightarrow(\mathcal{G})_{r}$.

2. The partite lemma. In this section we derive a key lemma for our further approach. Our method is based on the celebrated Hales-Jewett theorem which we recall here.

Let $A$ be a finite set. Consider the set $A^{n}$ of all $n$-tuples of elements of $A$. A combinatorial line $\mathcal{L} \subset A^{n}$ is any set of the form

$$
\mathcal{L}=\left\{\left(x_{1}, \ldots, x_{n}\right), x_{i}=x_{i^{\prime}} \text { for all } i, i^{\prime} \in I, x_{j}=b_{j} \text { for } j \notin I\right\},
$$

where $I$ is a fixed nonempty subset of $\{1,2, \ldots, n\}$ and $b_{j}, j \notin I$, a fixed sequence of elements of $A$.

With this terminology the Hales-Jewett theorem is very easy to state:

THEOREM 2.1 (SEE [5]). For all finite sets $A$ and every integer $r \geq 2$ there exists $n(A, r)$ such that for $n \geq n(A, r)$, in every $r$-coloring of $A^{n}$ there is a monochromatic line. 
We shall use the following

DEFINITION 2.2. Let $F \subset A^{n}, f=\left\{\bar{\nu}_{1}, \bar{\nu}_{2}, \ldots, \bar{\nu}_{p}\right\}$ and $t \leq n$. We set $\pi_{t}(F)=$ $\left\{\nu_{1 t}, \nu_{2 t}, \ldots, \nu_{p t}\right\}$ where $\bar{\nu}_{i}=\left\{\nu_{i 1}, \nu_{i 2}, \ldots, \nu_{i n}\right\}$. The set $\pi_{t}(F)$ is called the $t$ th projection of the set $F$. In the rest of this section we shall deal with $k$-partite $k$-uniform hypergraphs:

DEFINITION 2.3. Suppose that $\left.\left(\left(X_{j}\right)\right)_{j=1}^{k}, \mathcal{G}\right) \in \operatorname{Part}(k, l)$. Let $n$ be a positive integer. We define the $k$-partite hypergraph

$$
\left(\left(Y_{j}\right)_{j=1}^{k}, \mathcal{F}\right)=\left(\left(X_{j}\right)_{j=1}^{k}, \mathcal{G}\right)^{n}
$$

in the following way.

$$
Y_{j}=\prod_{t \leq n} X_{j}^{t}, \quad \text { where } X_{j}^{t} \text { are copies of the set } X_{j}, j \leq k
$$

and

$$
\mathcal{F}=\left\{F \subset \bigcup_{j=1}^{k} Y_{j}: \pi_{t}(F) \in \mathcal{G} \text { for every } t \leq n\right\} .
$$

Proposition 2.4. Suppose that $\left(\left(X_{j}\right)_{j=1}^{k}, \mathcal{G}\right) \in \operatorname{Part}(k, l), n$ is a positive integer. Then

$$
\left(\left(Y_{j}\right)_{j=1}^{k}, \mathcal{F}\right)=\left(\left(X_{j}\right)_{j=1}^{k}, \mathcal{G}\right)^{n} \in \operatorname{Part}(k, l) .
$$

ProOF. Suppose that there exist $F, F^{\prime} \in \mathcal{F}, F \neq F^{\prime},\left|F \cap F^{\prime}\right| \geq l$. Let $\nu_{1}, \nu_{2}, \ldots, \nu_{l} \in F \cap F^{\prime}, \nu_{i} \neq \nu_{j}$ for all $1 \leq i<j \leq l$. Then $\pi_{t}\left(\nu_{1}\right), \pi_{t}\left(\nu_{2}\right), \ldots, \pi_{t}\left(\nu_{l}\right)$ $\in \pi_{t}(F) \cap \pi_{t}\left(F^{\prime}\right)$ and hence also $\pi_{t}\left(\nu_{i}\right) \neq \pi_{t}\left(\nu_{j}\right)$ for all $1 \leq i<j \leq l$ and $t \leq n$. On the other hand, as $F \neq F^{\prime}$ there exists $t_{0} \leq n$ such that $\left|\pi_{t_{0}}(F) \cap \pi_{t_{0}}\left(F^{\prime}\right)\right|<l$, a contradiction.

DEFINITION 2.5. Let

$$
\left(\left(X_{i}\right)_{i=1}^{k}, \mathcal{G}\right) \in \operatorname{Part}(k, l) \quad \text { and } \quad\left(\left(Y_{i}\right)_{i=1}^{k}, \mathcal{F}\right)=\left(\left(X_{i}\right)_{i=1}^{k}, \mathcal{G}\right)^{n} .
$$

Let $\lambda: \mathcal{F} \rightarrow \mathcal{G}^{n}$ be a mapping defined by $\lambda(F)=\left(\pi_{1}(F), \pi_{2}(F), \ldots, \pi_{n}(F)\right)$. Clearly $\lambda$ is $1-1$ correspondence between $\mathcal{F}$ and $\mathcal{G}^{n}$. We say that the $k$-graph $\left(\left(Z_{j}\right)_{j=1}^{k}, \mathcal{H}\right)$ is a canonical subgraph of $\left(\left(Y_{j}\right)_{j=1}^{k}, \mathcal{F}\right)$ if the set $\{\lambda(H) ; H \in \mathcal{H}\}$ is a line and $\bigcup_{j=1}^{k} Z_{j}=\bigcup\{H ; H \in \mathcal{H}\}$.

Proposition 2.6. Let $\left(\left(X_{j}\right)_{j=1}^{k}, \mathcal{G}\right) \in \operatorname{Part}(k, l)$ and let $\left(\left(Z_{j}\right)_{j=1}^{k}, \mathcal{H}\right)$ be a canonical subgraph of

$$
\left(\left(X_{j}\right)_{j=1}^{k}, \mathcal{G}\right)^{n}=\left(\left(Y_{j}\right)_{j=1}^{k}, \mathcal{F}\right)
$$

Set

$$
\mathcal{H}=\left\{H_{1}, H_{2}, \ldots, H_{p}\right\}, \quad \mathcal{G}=\left\{G_{1}, G_{2}, \ldots, G_{p}\right\}, \quad \mathcal{F}=\left\{F_{1}, \ldots, F_{p^{n}}\right\} .
$$

Then the following holds:

(a) There exists a set $N=N(\mathscr{H}) \subset\{1,2, \ldots, n\}$ and $G_{i_{t}} \in \mathcal{G}, t \in\{1,2, \ldots, n\}-$ $N$ such that

$$
\pi_{t}\left(H_{j}\right)= \begin{cases}G_{i_{t}} & \text { for every } t \notin N \text { and every } j=1,2, \ldots, p, \\ G_{j} & \text { for every } t \in N \text { and } j=1,2, \ldots, p .\end{cases}
$$

(b) For every $\nu \in Z_{j}$ there exists $x \in X_{j}$ such that $\pi_{t}(\nu)=x$ for every $t \in N(\mathcal{H})$. The mapping $\nu \mapsto x$ is an isomorphism between $\left(\left(Z_{j}\right)_{j=1}^{k}, \mathcal{H}\right)$ and $\left(\left(X_{j}\right)_{j=1}^{k}, \mathcal{G}\right)$. 
(c) Let $\left\{\nu_{1}, \ldots, \nu_{k^{\prime}}\right\} \subset \bigcup_{j=1}^{k} Z_{j}$. Then for every $t \in\{1,2, \ldots, n\}-N(\mathcal{H})$ there exists $G \in \mathcal{G}$ with the property that $\left\{\pi_{t}\left(\nu_{1}\right), \pi_{t}\left(\nu_{2}\right), \ldots, \pi_{t}\left(\nu_{k^{\prime}}\right)\right\} \subset G$.

(d) Let $\nu_{1}, \nu_{2}, \ldots, \nu_{l} \in \bigcup_{j=1}^{k} Z_{j}$ and for every $t \leq n$ let there exist $G_{i_{t}} \in \mathcal{G}$ such that $\left\{\pi_{t}\left(\nu_{1}\right), \pi_{t}\left(\nu_{2}\right), \ldots, \pi_{t}\left(\nu_{l}\right)\right\} \subset G_{i_{t}}$. Then there exists $H \in \mathcal{H}$ such that $\pi_{t}(H)=G_{i_{t}}$ for every $t \leq n$.

ProOF. We prove (d) only. Let $t(1), t(2) \in N(\not{H})$. As $\left(\left(X_{j}\right)_{j=1}^{k}, \mathcal{G}\right) \in \operatorname{Part}(k, l)$ the edges $G_{i_{t(1)}}$ and $G_{i_{t(2)}}$ are determined uniquely and (because of 2.6(b)) $G_{i_{t(1)}}=$ $G_{i_{t(2)}}$. Thus the edge $H \in \mathcal{F}$ with $\pi_{t}(H)=G_{i_{t}}$ for every $t \leq n$ is an element of $\not H$ also.

Next we verify the following, intuitively clear statement.

LEMMA 2.7. Let $\left(\left(X_{j}\right)_{j=1}^{k}, \mathcal{G}\right) \in \operatorname{Part}(k, l)$ and let $n$ be a positive integer. Let $\left(\left(Z_{j}\right)_{j=1}^{k}, \forall\right)$ be a canonical subgraph of $\left(\left(X_{j}\right)_{j=1}^{k}, \mathcal{G}\right)^{n}$. Then

$$
\left(\left(Z_{j}\right)_{j=1}^{k}, \not H\right) \cong\left(\left(X_{j}\right)_{j=1}^{k}, \mathcal{G}\right) \text {. }
$$

PROOF. Let $\mathcal{G}=\left\{G_{1}, G_{2}, \ldots, G_{p}\right\}$. Take the sets $G_{i_{t}}$ with the property of Proposition 2.6(a) and define the mapping $\phi\left(\left(X_{j}\right)_{j=1}^{k}, \mathcal{G}\right) \rightarrow\left(\left(Z_{j}\right)_{j=1}^{k}, \mathcal{H}\right)$ in the following way: for $x \in X_{j}, j \leq k$, set

$$
\phi(x)=\left(a_{1}, a_{2}, \ldots, a_{n}\right)
$$

where

$$
a_{t}= \begin{cases}G_{i_{t}} \cap X_{j} & \text { for } t \notin N(\mathcal{H}), \\ x & \text { for } t \in N(\not{H}) .\end{cases}
$$

Clearly, $\phi$ is bijective. We prove that $\phi$ is an isomorphism. Let $G_{j}=\left\{x_{1}, x_{2}, \ldots, x_{k}\right\}$ $\in \mathcal{G}, x_{i} \in X_{i}$ for $i \leq k$. Then

$$
\pi_{t}\left\{\phi\left(x_{1}\right), \phi\left(x_{2}\right), \ldots, \phi\left(x_{k}\right)\right\}= \begin{cases}G_{i_{t}} & \text { for } t \notin N(\not{H}), \\ G_{j} & \text { for } t \in N(\not{H})\end{cases}
$$

and thus $\left\{\phi\left(x_{1}\right), \phi\left(x_{2}\right), \ldots, \phi\left(x_{k}\right)\right\} \in \not{K}$. On the other hand if $\left\{x_{1}, x_{2}, \ldots, x_{k}\right\} \notin \mathcal{G}$ then clearly

$$
\pi_{t}\left\{\phi\left(x_{1}\right), \ldots, \phi\left(x_{k}\right)\right\}=\left\{x_{1}, x_{2}, \ldots, x_{k}\right\} \notin \mathcal{G} \quad \text { for any } t \in N(\mathscr{H})
$$

and thus $\left\{\phi\left(x_{1}\right), \ldots, \phi\left(x_{k}\right)\right\} \notin \not$.

LEMMA 2.8. Let $\left(\left(X_{j}\right)_{j=1}^{k}, \mathcal{G}\right) \in \operatorname{Part}(k, l)$ and let $\left(\left(Z_{j}^{1}\right)_{j=1}^{k}, \not^{1}\right),\left(\left(Z_{j}^{2}\right)_{j=1}^{k}, \not^{2}\right)$ be two different canonical subgraphs of $\left(\left(X_{j}\right)_{j=1}^{k}, \mathcal{G}\right)^{n}$ such that there exists l vertices $\nu_{1}, \nu_{2}, \ldots, \nu_{l} \in \bigcup_{j=1}^{k} Z_{j}^{1} \cap \bigcup_{j=1}^{k} Z_{j}^{2}$ with the property

$$
\left|\left\{\nu_{1}, \nu_{2}, \ldots, \nu_{l}\right\} \cap Z_{j}^{1} \cap Z_{j}^{2}\right| \leq 1 \quad \text { for all } j \leq k .
$$

Then there exists $E \in \mathfrak{H}^{1} \cap \mathcal{H}^{2}$ such that $\left\{\nu_{1}, \nu_{2}, \ldots, \nu_{l}\right\} \subset E$.

PROOF. Suppose that there exist $\nu_{1}, \nu_{2}, \ldots, \nu_{l} \in \bigcup_{j=1}^{k} Z_{j}^{1} \cap \bigcup_{j=1}^{k} Z_{j}^{2}, \nu_{i} \neq \nu_{i}^{\prime}$, for $i \neq i^{\prime}$ such that

(i) $\left|\left\{\nu_{1}, \nu_{2}, \ldots, \nu_{l}\right\} \cap Z_{j}^{1} \cap Z_{j}^{2}\right| \leq 1$ for all $j \leq k$,

(ii) $\left\{\nu_{1}, \nu_{2}, \ldots, \nu_{l}\right\} \not \subset E$ for all $E \in \not^{1} \cap \not^{2}$.

With respect to (ii) there exsits $t_{0} \leq n$ such that (*) $\left\{\pi_{t_{0}}\left(\nu_{1}\right), \ldots, \pi_{t_{0}}\left(\nu_{l}\right)\right\} \not \subset G \quad$ for all $G \in\left\{\pi_{t_{0}}(H) ; H \in \not^{1}\right\} \cap\left\{\pi_{t_{0}}(H) ; H \in \not{H}^{2}\right\}$. 
Clearly, $\left\{\pi_{t_{0}}(H) ; H \in \mathcal{H}^{i}\right\}, i=1,2$, is either a one-element set or equals the set G. Therefore we get one of the subsequent cases:

$(\alpha)$ Either $\left\{\pi_{t_{0}}(H), H \in \mathcal{H}^{1}\right\}$ or $\left\{\pi_{t_{0}}(H), H \in \mathscr{H}^{2}\right\}$ are one-element sets or

(B) $\left\{\pi_{t_{0}}(H) ; H \in \mathscr{H}^{1}\right\}=\left\{\pi_{t_{0}}(H) ; H \in \mathscr{H}^{2}\right\}=\mathcal{G}$.

Suppose first that $\left\{\pi_{t_{0}}(H) ; H \in \mathcal{H}^{1}\right\}=\left\{G^{1}\right\}$, where $G^{1} \in \mathcal{G}$. This implies that $\left\{\pi_{t_{0}}\left(\nu_{1}\right), \ldots, \pi_{t_{0}}\left(\nu_{l}\right)\right\} \subset G^{1}$. Hence according to $(*) G^{1} \notin\left\{\pi_{t_{0}}(H), H \in\right.$ $\left.\mathscr{H}^{2}\right\}$ and thus the set $\left\{\pi_{t_{0}}(H), H \in \mathcal{H}^{2}\right\}$ consists of a single element, we denote it by $G^{2}$. Again, by $(*)$ we get that $G^{1} \neq G^{2}$ and hence $l>\left|G^{1} \cap G^{2}\right| \geq$ $\left|\left\{\pi_{t_{0}}\left(\nu_{1}\right), \ldots, \pi_{t_{0}}\left(\nu_{l}\right)\right\}\right|=l$, a contradiction. Suppose now that $(\beta)$ holds. Thus $\left\{\pi_{t_{0}}\left(\nu_{1}\right), \ldots, \pi_{t_{0}}\left(\nu_{l}\right)\right\} \not \subset G$ for all $G \in \mathcal{G}$ and hence $t_{0} \in N\left(\mathcal{H}^{1}\right) \cap N\left(\not^{2}\right)$. Since (according to $2.6(\mathrm{~b})) \pi_{t}\left(\nu_{1}\right)=\pi_{t_{0}}\left(\nu_{1}\right), \pi_{t}\left(\nu_{2}\right)=\pi_{t_{0}}\left(\nu_{2}\right), \ldots, \pi_{t}\left(\nu_{l}\right)=\pi_{t_{0}}\left(\nu_{l}\right)$ for all $t \in N\left(\mathcal{H}^{1}\right) \cup\left(\mathcal{H}^{2}\right)$ we get that $\left\{\pi_{t}\left(\nu_{1}\right), \pi_{t}\left(\nu_{2}\right), \ldots, \pi_{t}\left(\nu_{l}\right)\right\} \not \subset G$ for all $G \in \mathcal{G}$ and $t \in N\left(\mathscr{H}^{1}\right) \cup N\left(\mathscr{H}^{2}\right)$. According to 2.6(c) $t \notin N\left(\mathcal{H}_{1}\right)\left(t \notin N\left(\mathcal{H}_{2}\right)\right)$ implies $t \notin N\left(\mathscr{H}^{1}\right) \cup N\left(\not{H}^{2}\right)$ and hence $N\left(\mathscr{H}^{1}\right)=N\left(\not{H}^{2}\right)$.

As $\left(\left(Z_{j}^{1}\right)_{j=1}^{k}, \mathfrak{H}^{1}\right) \neq\left(\left(Z_{j}^{2}\right)_{j=1}^{k}, \mathfrak{H}^{2}\right)$ and $N\left(\mathfrak{H}^{1}\right)=N\left(\mathfrak{H}^{2}\right)$ we get that there exists $s \in\{1,2, \ldots, n\}-N\left(\mathcal{H}^{1}\right)$ such that

$$
\begin{aligned}
& \pi_{s}\left(\bigcup_{j=1}^{k} Z_{j}^{1}\right)=G_{1} \in \mathcal{G}, \\
& \pi_{s}\left(\bigcup_{j=1}^{k} Z_{1}^{2}\right)=G_{2} \in \mathcal{G}, \quad \text { where } G_{1} \neq G_{2} .
\end{aligned}
$$

Hence

$$
l>\left|G_{1} \cap G_{2}\right| \geq\left|\pi_{s}\left(\bigcup_{j=1}^{k} Z_{j}^{1} \cap \bigcup_{j=1}^{k} Z_{j}^{2}\right)\right| \geq\left|\left\{\pi_{s}\left(\nu_{1}\right), \ldots, \pi_{s}\left(\nu_{l}\right)\right\}\right|=l
$$

a contradiction.

Lemma 2.9 (PARTite Lemma). Let $\left(\left(X_{j}\right)_{j=1}^{k}, \mathcal{G}\right) \in \operatorname{Part}(k, l)$ and let $r$ be a positive integer. Then there exists a positive integer $n$ such that the hypergraph $\left(\left(Y_{j}\right)_{j=1}^{k}, \mathcal{F}\right)=\left(\left(X_{j}\right)_{j=1}^{k}, \mathcal{G}\right)^{n}$ has the following property:

For every partition $\mathcal{F}=\mathcal{F}_{1} \cup \mathcal{F}_{2} \cup \cdots \cup \mathcal{F}_{r}$ there exists $i \leq r$ and a canonical subgraph $\left(\left(Z_{j}\right)_{j=1}^{k}, \mathcal{H}\right)$ such that $\mathcal{H}=\mathcal{F}_{i}$.

ProOF. Follows immediately from Hales-Jewett theorem, using the correspondence between $\mathcal{F}$ and $\mathcal{G}^{n}$ described in 2.5 .

3. Systems of copies. Let $S(k, l)$ be the system of all partial Steiner $(k, l)$ systems and let $\mathcal{G}, \mathcal{H} \in S(k, l)$. We define an $|\mathcal{E}(\mathcal{G})|$-uniform hypergraph $\left(\mathcal{E}(\mathfrak{H}), \mathcal{E}_{\mathcal{G}}^{\mathcal{H}}\right)$ in the following way: vertices of $\left(\mathcal{E}(\mathcal{H}), \mathcal{E}_{\mathcal{G}}^{\mathcal{H}}\right)$ are the edges of $\not{H}$ and $T \in \mathcal{E}_{\mathcal{G}}^{\not H}$ iff $(\bigcup T, T) \in\left(\begin{array}{l}\mathcal{H} \\ g\end{array}\right)$, more explicitly $T \in \mathcal{E}_{g}^{H}$ if $T$ is a set of edges of induced subgraph of $\not{H}$ which is isomorphic to $\mathcal{G}$. Clearly $\mathscr{H} \rightarrow(\mathcal{G})_{r}$, if and only if $\chi\left(\mathcal{E}(\mathcal{H}), \mathcal{E}_{\mathcal{G}}^{\mathcal{H}}\right)>r$, where $\chi$ denotes the chromatic number. 
DEFinition 3.1. Let $\mathcal{G}, \not{H} \in S(k, l)$ (resp. Part $(k, l))$. An arbitrary set $S \subset\left(\begin{array}{l}\not{H} \\ \mathcal{G}\end{array}\right)$ is called a system of copies of $\mathcal{G}$ in $\not$. Let $r$ be a positive integer. We say that the system of copies $S$ is $r$-Ramsey for $\mathcal{G}$ if for any partition $\mathcal{E}(\mathcal{H})=\mathcal{E}_{1} \cup \mathcal{E}_{2} \cup \cdots \cup \mathcal{E}_{r}$ there exists an induced subgraph $\mathcal{G}^{\prime} \leq \mathcal{H}$ such that

(i) $\mathcal{G}^{\prime} \in S$,

(ii) $\mathcal{E}\left(\mathcal{G}^{\prime}\right) \subseteq \mathcal{E}_{j}$ for some $j \leq r$.

This fact we abbreviate $S \rightarrow(\mathcal{G})_{r}$.

DEFINITION 3.2. Let $r$ be a positive integer and $\mathcal{G} \in \operatorname{Part}(k, l)$. Let $n$ be a minimal positive integer such that the hypergraph $\mathcal{G}^{n}$ has the following property: if we set $S=\left\{F, F\right.$ is a canonical subgraph of $\left.\mathcal{G}^{n}\right\}$, then $S \rightarrow(\mathcal{G})_{r}$. Next we shall denote by $R_{r}(\mathcal{G})$ the hypergraph $\mathcal{G}^{n}$ and by $S_{r}(\mathcal{G})$ the system $S$.

4. The partite amalgamation. In this section we recall the partite amalgamation which we already used in $[\mathbf{1 0}, \mathbf{1 1}]$.

CONSTRUCTION 4.1. Let $\mathcal{G} \in S(k, l)$ and let $K_{m}^{k}$ be a complete $k$-uniform hypergraph with $m$ vertices. Let

$$
\left[\begin{array}{c}
K_{m}^{k} \\
\mathcal{g}
\end{array}\right]=\left\{\mathcal{g}_{1}, g_{2}, \ldots, g_{p}\right\}
$$

be a system of subgraphs of $K_{m}^{k}$ (not necessarily induced) which are isomorphic to 9. According to the well-known Ramsey theorem $[\mathbf{5}, \mathbf{1 4}]$ there exists $m$ such that for every partition of the edges of $K_{m}^{k}$ into $r$ classes $\varepsilon_{1} \cup \mathcal{E}_{2} \cup \cdots \cup \mathcal{E}_{r}$ there exists $i, 1 \leq i \leq r$, such that $\mathcal{E}\left(\mathcal{G}_{i}\right) \subset \mathcal{E}_{i}$ for some $j, 1 \leq j \leq p$. Next we fix $m$ with this property: Set

$$
V\left(K_{m}^{k}\right)=\left\{\nu_{1}, \nu_{2}, \ldots, \nu_{m}\right\}, \quad \mathcal{E}\left(K_{m}^{k}\right)=\left\{E_{1}, E_{2}, \ldots, E_{\left(\begin{array}{c}
m \\
k
\end{array}\right)}\right\} .
$$

We define inductively the $m$-partite $k$-graph $\rho\left(\begin{array}{c}m \\ k\end{array}\right)=\not k * \mathcal{G}$ in the following way:

I. Set $p^{0}=\left(\left(V_{i}^{0}\right)_{i=1}^{m}, \mathcal{E}^{0}\right)$ where $V_{i}^{0}=\left\{\left(\nu_{i}, j\right), j \leq p, \nu_{i} \in V\left(\mathcal{G}_{j}\right)\right\}$ and $\left\{\left(\nu_{i_{1}}, j_{1}\right),\left(\nu_{i_{2}}, j_{2}\right), \ldots,\left(\nu_{i_{k}}, j_{k}\right)\right\} \in \mathcal{E}^{0}$ if and only if $j_{1}=j_{2}=\cdots=j_{k}=j$ and $\left\{\nu_{i_{1}}, \nu_{i_{2}}, \ldots, \nu_{i_{k}}\right\} \in \mathcal{E}\left(G_{j}\right)$.

II. Suppose we defined $r$-partite $k$-uniform hypergraphs $\rho^{s}=\left(\left(V_{i}^{s}\right)_{i=1}^{m}, \mathcal{E}^{s}\right)$. Let $E_{s+1}=\left\{u_{x 1}, u_{x 2}, \ldots, u_{x k}\right\}$ and let $\mathcal{F}_{s}$ be a $k$-partite subgraph of $\rho^{s}$ induced on the set $\bigcup_{i=1}^{k} V_{x_{i}}^{s}$. Let $S_{r}\left(\mathcal{F}_{s}\right)=\left\{\mathcal{F}_{s}^{1}, \mathcal{F}_{s}^{2}, \ldots, \mathcal{F}_{s}^{q}\right\}$ and let $\phi_{j}: \mathcal{F}_{s} \rightarrow \mathcal{F}_{s}^{j}, j=1,2, \ldots, q$ be an isomorphism. Set $V\left(p^{s+1}\right)=\bigcup_{i=1}^{m} V_{i}^{s+1}$ where

$$
\begin{aligned}
& V_{i}^{s+1}=\bigcup_{j \leq q} V_{i}^{s} \times\{j\} \quad \text { for } i \notin\left\{x_{1}, x_{2}, \ldots, x_{k}\right\}, \\
& V_{x_{i}}^{s+1}=V_{i}\left(R_{r}\left(\mathcal{F}_{s}\right)\right), \quad i \in\{1,2, \ldots, k\} .
\end{aligned}
$$

Denote by $\psi_{j}: V\left(\rho^{s}\right) \rightarrow V\left(p^{s+1}\right)$ the mapping defined by

$$
\psi_{j}(\nu)= \begin{cases}\phi_{j}(\nu) & \text { for } \nu \in V\left(\mathcal{F}_{s}\right), \\ (\nu, j) & \text { for } \nu \notin V\left(\mathcal{F}_{s}\right) .\end{cases}
$$

Set $\left\{\nu_{1}, \nu_{2}, \ldots, \nu_{k}\right\} \in \mathcal{E}\left(P^{s+1}\right)$ if and only if there exist $j \leq q$ and $\left\{u_{1}, u_{2}, \ldots, u_{k}\right\} \in$ $\mathcal{E}\left(P^{s}\right)$ so that $\nu_{1}=\psi_{j}\left(u_{1}\right), \nu_{2}=\psi_{j}\left(u_{2}\right), \ldots, \nu_{k}=\psi_{j}\left(u_{k}\right)$. 
The following is an immediate consequence of the construction:

Proposition 4.2. Let $\overline{\mathcal{P}}_{1}^{s}: \overline{\mathcal{P}}_{2}^{s} \in\left(\begin{array}{c}\mathcal{P}^{s+1} \\ \rho^{s}\end{array}\right), \overline{\mathcal{P}}_{1}^{s} \neq \overline{\mathcal{P}}_{2}^{s}$. Then $V_{i}\left(\overline{\mathcal{P}}_{1}^{s}\right) \cap V_{i}\left(\mathcal{P}_{2}^{s}\right)=0$ for all $i \in\{1,2, \ldots, r\}-\left\{x_{1}, \ldots, x_{k}\right\}$.

The next proposition is easy; for the proof see e.g. [10].

PROPOSITION 4.3. $\rho\left(\begin{array}{c}m \\ k\end{array}\right) \rightarrow(\mathcal{G})_{r}$.

5. Proof of Theorem 1.1. Let $\mathcal{G} \in S(k, l)$. We apply the above construction and set $P\left(\begin{array}{c}m \\ k\end{array}\right)=\mathscr{H}$. According to Proposition 4.3 it remains to prove only that $\sharp$ is a Steiner $(k, l)$-system.

Suppose that $P_{s} \in S(k, l)$, according to Lemma 2.8 and Proposition 4.2, for any two copies $\bar{P}_{1}^{s}, \bar{P}_{2}^{s} \in\left(\begin{array}{c}P^{s+1} \\ p_{s}\end{array}\right)$ the intersection $V\left(\bar{P}_{1}^{s}\right) \cap V\left(\bar{P}_{2}^{s}\right)$ is either an edge of $p^{s+1}$ or has an empty intersection with all but at most $l^{\prime}<l$ of the classes $V_{i}^{s+1}$, $i=1,2, \ldots, m$. This, however, implies that $p^{s+1} \in S(k, l)$.

6. Application to bipartite graphs. One of the important questions of structural Ramsey theory is to characterize the classes of graphs with edge partition property. The first unsolved problem here was whether the class of bipartite $C_{4^{-}}$ free graphs has the edge partition property. The obstacle in solving this question was that all "natural" (and known) constructions of Ramsey graphs were using set representations, products and other techniques that were not able to exclude the existence of $C_{4}$ or more generally complete bipartite graphs. This together with some other aspects (certain analogues between partition properties of finite and infinite graphs) led to the conjecture that the class of graphs with girth at least five does not have the edge partition property [3]. This conjecture can be disproved using our approach. First we establish the edge partition property of the class of bipartite $C_{4}$-free graphs.

DEFINITION 6.1. The ordered hypergraph $(\mathcal{G}, \leq)$ is a pair, where $\mathcal{G}$ is a hypergraph and $\leq$ is linear ordering of its vertices. Let $(\mathcal{G}, \leq),(\mathcal{H}, \leq)$ be two ordered hypergraphs, $\mathcal{G}=(V, \mathcal{E}), \quad \mathscr{H}=(W, \mathcal{F})$. We say that the mapping $\phi: V(\mathcal{G}) \rightarrow V(\mathcal{H})$ is an ordered embedding if $\phi(\nu)<\phi\left(\nu^{\prime}\right)$ for $\nu<\nu^{\prime}$ and $\left\{\phi\left(\nu_{1}\right), \phi\left(\nu_{2}\right), \ldots, \phi\left(\nu_{k}\right)\right\} \in \mathcal{F}$ if and only if $\left\{\nu_{1}, \nu_{2}, \ldots, \nu_{k}\right\} \in \mathcal{E}$.

The proof of Theorem 1.1 yields also the following slightly stronger result.

THEOREM 6.2. Let $(\mathcal{G}, \leq)$ be an ordered $k$-uniform hypergraph. $\mathcal{G} \in S(k, l)$ and let $r \geq 2$ be a positive integer. Then there exists an ordered $k$-uniform hypergraph $(\mathcal{H}, \leq)$, $\mathcal{H} \in S(k, l)$ such that for every partition $\mathcal{E}(\not{H})=\mathcal{E}_{1} \cup \mathcal{E}_{2} \cup \cdots \cup \mathcal{E}_{r}$ there exists $i \leq r$ and an ordered embedding $\phi(\mathcal{G}, \leq) \rightarrow(\mathcal{H}, \leq)$ such that $\phi(\mathcal{E}(\mathcal{G})) \subseteq \mathcal{E}_{i}$. This fact will be denoted by $(\mathcal{H}, \leq) \rightarrow(\mathcal{G}, \leq)_{r}$.

Now we are ready to prove

THEOREM 6.3. The class of all bipartite graphs without cycle of length four has the edge partition property.

ProOF. Let $\mathcal{G}=\left(U, U^{\prime}, \mathcal{E}\right)$ be a bipartite graph and $r \geq 2$ a positive integer. Without loss of generality assume that $d(u)=d$ for every $u \in U^{\prime}$. For $u \in U^{\prime}$ define $M_{u}=\{\nu \in U ;\{u, \nu\} \in \mathcal{E}\}$ and $\mathcal{M}=\left\{M_{u}: u \in U^{\prime}\right\}$. Then $(U, \mathcal{M})$ is a $d$-uniform hypergraph. As $\left(U, U^{\prime}, \mathcal{E}\right)$ does not contain $C_{4}$ we have that $(U, \mathcal{M}) \in S(d, 2)$. Set 
$k=r(d-1)+1$ and let $(V, \mathcal{N}) \in S(k, 2)$ be a $k$-graph which has the following property.

There exists an ordering $\leq_{1}$ of the set $V$ such that, for every $K \subset\{1,2, \ldots, k\},|K|=d$, the hypergraph $(U, \mathcal{M})$ is an in(*) duced subgraph of $\left(V, \mathcal{N}_{K}\right)$, defined by $\mathcal{N}_{K}=\left\{N_{k}, N \in \mathcal{N}\right\}$, where $N_{K}=\left\{\nu ; \nu\right.$ is the $i$ th element of $N$ (with respect to $\leq_{1}$ ) where $i \in K\}$.

Set $r^{\prime}=r^{k}$. Let $\left((W, \mathcal{L}), \leq_{2}\right)$ be an ordered $k$-uniform hypergraph, such that $(W, \mathcal{L}) \in S(k, 2)$ and $\left((W, \mathcal{L}), \leq_{2}\right) \rightarrow\left((V, \mathcal{N}), \leq_{1}\right)_{r}$.

We define the bipartite graph $(W, \mathcal{L}, \mathcal{F})$ in the following way: $\{w, E\} \in \mathcal{F}$ iff $w \in E$.

We prove $(W, \mathcal{L}, \mathcal{F}) \rightarrow\left(U^{\prime}, U, \mathcal{E}\right)_{r}$. Let $\mathcal{F}=\mathcal{F}_{1} \cup \mathcal{F}_{2} \cup \cdots \cup \mathcal{F}_{r}$ be a partition. For every $E \in \mathcal{L}$ this defines a mapping $\phi: E \rightarrow\{1,2, \ldots, r\}$ defined by $\phi(w)=j$ if and only if $\{w, E\} \in \mathcal{F}_{j}$. Let $\mathcal{L}=\mathcal{L}_{1} \cup L_{2} \cup \cdots \cup \mathcal{L}_{r^{\prime}}$, be the partition defined in the following way: $\left\{\nu_{1}, \nu_{2}, \ldots, \nu_{k}\right\}, \nu_{1} \leq \nu_{2} \leq \cdots \leq \nu_{k}$, is in the same partition class as $\left\{w_{1}, w_{2}, \ldots, w_{k}\right\}, w_{1} \leq w_{2} \leq \cdots \leq w_{k}$, iff $\phi\left(\nu_{j}\right)=\phi\left(w_{j}\right)$ for all $j \leq k$. According to $(* *)$ there exists an ordered embedding $\phi:\left((V<\mathcal{N}), \leq_{1}\right) \rightarrow\left((W, \mathcal{L}), \leq_{2}\right)$ such that $\phi(\mathcal{N}) \subset \mathcal{L}_{i}$ for some $i \leq r^{\prime}$. Set $\bar{V}=\phi(V)$ and let $(\bar{V}, \overline{\mathcal{N}})$ be a subgraph of $(W, \mathcal{L})$ induced on $\bar{V}$. Let $(\bar{V}, \overline{\mathcal{N}}, \mathcal{M})$ be a bipartite graph defined by $\{\nu, N\} \in \mathcal{M}$ if and only if $\nu \in \overline{\mathcal{N}} .(\bar{V}, \overline{\mathcal{N}}, \mathcal{M})$ is clearly an induced subgraph of $(W, \mathcal{L}, \mathcal{F})$. Moreover, we have for every $N, M \in \overline{\mathcal{N}}, N=\left\{\nu_{1}, \ldots, \nu_{k}\right\}, M=\left\{w_{1}, \ldots, w_{k}\right\}$ that $\left\{\nu_{i}, N\right\} \in \mathcal{F}_{j}$ if and only if $\left\{w_{i}, M\right\} \in \mathcal{F}_{j}$. As $k=r(d-1)+1$ there exists $K \subset\{1,2, \ldots, k\},|K|=d$, and $j_{0} \leq r$ such that for every $N=\left\{\nu_{1}, \ldots, \nu_{k}\right\} \in \overline{\mathcal{N}}$ we have $\left\{\nu_{i}, N\right\} \in \mathcal{F}_{j_{0}}$ for all $i \in K$. According to $(*)$ we get that there exists $\left(\bar{U}, \bar{U}^{\prime}, \overline{\mathcal{E}}\right) \cong\left(U, U^{\prime}, \mathcal{E}\right)$ which is an induced subgraph of both $(\bar{V}, \overline{\mathcal{N}}, \mathcal{M})$ and $\left(W, \mathcal{L}, \mathcal{F}_{j_{0}}\right)$.

7. Application to graphs with girth larger than four. We start with some discussion of the proof of Theorem 1.1. Note that for $\mathcal{G} \in S(k, 2)$ the hypergraph $\forall \in S(k, 2)$ constructed in Theorem 1 . has also the following property.

For any two $\mathcal{G}_{1}, \mathcal{G}_{2} \in\left(\begin{array}{l}\mathcal{H} \\ g\end{array}\right)$ either

$$
V\left(\mathcal{G}_{1}\right) \cap V\left(\mathcal{G}_{2}\right) \subset E \quad \text { for some } E \in \mathcal{E}(\mathcal{H})
$$

or

$$
\left|V\left(\mathcal{G}_{1}\right) \cap V\left(\mathcal{G}_{2}\right)\right| \leq 1
$$

This can be proved by induction (similarly as we proved that $\not \in S(k, 2)$ ), using Lemma 2.8. Using this fact along the proof of Theorem 6.3 one can strengthen this theorem in the following way.

THEOREM 7.1. For every $C_{4}$-free bipartite graph $\mathcal{G}=(V, \mathcal{E})$ there exist a $C_{4}$ free bipartite graph $\mathcal{H}=(W, \mathcal{F})$ and a system $\mathcal{S}$ of copies of $\mathcal{G}$ in $\mathcal{H}$ such that

(i) $S \rightarrow(\mathcal{G})_{r}$ and

(ii) either $\left|V\left(\mathcal{G}_{1}\right) \cap V\left(\mathcal{G}_{2}\right)\right| \leq 1$ or $V\left(\mathcal{G}_{1}\right) \cap V\left(\mathcal{G}_{2}\right)$ is a star. 
Now we are ready to prove

THEOREM 7.2. The class of all graphs with girth at least five has edge partition property.

PROOF. We give a sketch only as the proof is based on the partite amalgamation discussed in $\S 4$. Let $\mathcal{G}$ be a graph with girth at least five, $r$ a given positive integer and let

$$
\left[\begin{array}{c}
K_{m}^{2} \\
\mathcal{G}
\end{array}\right]=\left\{\mathcal{G}_{1}, \mathcal{G}_{2}, \ldots, \mathcal{G}_{p}\right\}
$$

be a system of subgraphs of $K_{m}^{2}$ (not necessarily induced) which are isomorphic to G. Again, we take $m$ large so that for any partition of edges of $K_{m}^{2}$ into $r$ classes we get that all edges of $\mathcal{G}_{i}$ are in one of the classes. We copy Construction 4.1 with the following exception: as the graph $R_{r}(\mathcal{G})$ and system $S_{r}(\mathcal{G})$ we take the graph $\mathcal{H}$ and system $S$ from Theorem 7.1. We have again $\mathcal{P}\left(\begin{array}{c}m \\ 2\end{array}\right) \rightarrow(\mathcal{G})_{r}$. The only thing which remains to be checked is that $P\left(\begin{array}{c}m \\ 2\end{array}\right)$ has girth at least five. This, however, follows by induction as $\rho^{0}$ is certainly such and from the fact that $\rho^{s}$ has girth at least five we infer that $P^{s+1}$ is such as no cycle can be disconnected by the omission of a single point or a star.

REMARK. Note that five in Theorem 7.2 can be with the same proof replaced by six. We have more complicated argument for seven; however the present method does not allow us to go further.

\section{REFERENCES}

1. F. G. Abramson and L. A. Harrington, Models without indiscernibles, J. Symbolic Logic 43 (1978), 572-600.

2. W. Deuber, Generalizations of Ramsey's theorem, Colloq. Math. Soc. János Bolyai 10, NorthHolland, 1975 pp. 323-332.

3. P. Erdös, Problems and results on finite and infinite graphs, Recent Advances in Graph Theory, Academic Press, pp. 183-192.

4. P. Erdös, A. Hajnal and L. Pósa, Strong embeddings of graphs into colored graphs, Colloq. Math. János Bolyai Soc. 10, North-Holland, 1975, pp. 1127-1132.

5. R. L. Graham, Rudiments of Ramsey theory, CBMS Regional Conf. Ser. in Math., no. 45, Amer. Math. Soc., Providence, R. I., 1981.

6. J. Nešetřil and V. Rödl, Ramsey graph without triangles exists for any graph without triangles, Colloq. Math. Soc. János Bolyai 10, North-Holland, 1975, pp. 1127-1132.

7. _ Type theory of partition problems of graphs, Recent Advances in Graph Theory, Academic Press, pp. 405-412.

8. _ Ramsey property of graphs with forbidden complete subgraphs, J. Combin. Theory Ser. B 20, (1976), 243-249.

9. __ On Ramsey graphs without cycles of short odd length, Comment Math. Univ. Carolin. 29 (1979), 565-582.

10. 3 (1982), 347-352.

11. Simple proof of the existence of restricted Ramsey graphs by means of a partite construction, Combinatorica 1 (1982), 199-202.

12. $\ldots$, A short proof of the existence of highly chromatic hypergrahs without short cycles, J. Combin. Theory Ser. B 27(1979), 225-227. 
13. H. J. Prömel and B. Voigt, Canonizing Ramsey theory (preprint 1985).

14. F. P. Ramsey, On a problem of formal logic, Proc. London Math. Soc. 30 (1930), 264-286.

15. V. Rödl, A generalization of Ramsey theorem and dimension of graphs, Thesis, Charles University, 1973 (in Czech); English transl., A generalization of Ramsey theorem, Graphs, hypergraphs and block systems, (Eds., M. Borowiecki, Z. Skupien, and L. Szamkolowicz), Zielona Gora, 1976, pp. 211-220.

Department of Mathematics, AT\&T Bell laboratories, Murray hill, New JERSEY 07974

Department of Mathematics, Czech TeChnical University, Husova 5, 11000 Praha 1, Czechoslovakia (Current address of V. Rödl) 